Trauma Berufskrankh 2008 10 [Suppl 3]:408-410

DOI 10.1007/s10039-008-1412-y

Online publiziert: 29. Mai 2008

(c) Springer Medizin Verlag 2008

H. Belzl

Schule für Physiotherapie/Abteilung Physiotherapie, BG Unfallklinik, Tübingen

\title{
Physiotherapie nach operativer Stabilisierung von Wirbelsäulenverletzungen
}

ständigkeit im Alltag und Beruf wieder erreicht werden.

\section{Behandlungsplanung}

Neben den allgemeinen Prophylaxen folgt der frühfunktionelle Therapieansatz der Vorgabe, unter Berücksichtigung der verletzen Strukturen die Mobilität aufzubauen und zügig in die Koordinationskomplexe des Alltags einzubinden. Als grundsätzliche Aufgaben der Physiotherapie ergeben sich daraus:

- frühzeitige Mobilisation des Verletzen mit aktiver Stabilisierung der Wirbelsäule

- Vermittlung von Alltagsbewegungen mit dynamisch stabilisierter Wirbelsäule

- Aufbau der Selbständigkeit im Alltag

Wichtige Voraussetzungen hierfür sind umfassende Informationen des Operateurs zu den Fragen:

- erlaubtes Bewegen der Wirbelsäule

- erlaubtes Bewegen der Hüftgelenke

- Korsett oder Miederversorgung

chernen Statik an. Sie erfolgen bei:

- Frakturen mit Beteiligung der Wirbelhinterkante

- starker Deformierung des Wirbelkörpers

- neurologischer Symptomatik

Die Physiotherapie verfolgt in der konservativen sowie postoperativen Behandlung das Leitziel, die aktive Aufrichtung, die posturale Steuerung und Beweglichkeit des Rumpfs und der Wirbelsäule wiederherzustellen. Die Funktionen sollen in Alltagsbewegungen integriert, die Selbst-
Die dorsal instrumentierte Wirbelsäule ist in der Regel sofort bewegungsstabil, nach Abschluss der Wundheilung ist sie in Grenzen belastungsstabil. In der Regel erfolgt eine korsettfreie Nachbehandlung, eine Miederversorgung wird in Ausnahmefällen, z. B. bei älteren unsicheren Patienten oder Patienten mit Osteoporose, vorgenommen.

Derartige Behandlungspfade, wie sie in unserem Haus vorliegen, erlauben eine rasche und zielgerichtete Therapie. Ei- ne gemeinsame Strategie der Behandlung zwischen Operateur und Physiotherapie ist bei rückläufigen Liegezeiten für einen effektiven Therapieverlauf zwingend erforderlich.

Die physiotherapeutische Befunderhebung erfasst folgende Kriterien:

- Schmerz

- Schwellung

- Reduzierte Beweglichkeit

- Reflektorische Schutzschaltungen

- Angst

- Darmtätigkeit

Abhängig vom jeweiligen Ausprägungsgrad ergeben sich daraus die Behandlungsschwerpunkte:

- Schmerzlinderung

- Abschwellung

- Dynamische Stabilisation

- Training unverletzter Strukturen/Systeme

- Koordination

- Beweglichkeit

- Belastungssteigerung

\section{Therapie}

Trotz Behandlungspfaden sind die individuellen Befundergebnisse Grundlage einer gezielten, funktionsbezogenen und schonenden Physiotherapie. Der Aspekt der Anleitung zum eigenständigen Arbeiten ist im Therapiekonzept integriert.

Am Anfang steht die Bewusstmachung der Haltung und der Position des Rumpfs und der Wirbelsäule. Diese kann auch unter Spiegelkontrolle geschult werden. Distanzpunkte wie Symphyse, Bauchnabel, Sternumspitze und Kinnspitze helfen, ei- 
ne korrekte Sitz- und Standhaltung auch ohne Therapeut eigenständig einnehmen zu können.

Beim Training der wirbelsäulen- und rumpfumgebenden Muskulatur in verschiedenen Ausgangsstellungen wie Rückenlage, Bauchlage, hoher Sitz, Stand und Vierfüßlerstand stehen die dynamische Stabilisation, die Kraft und Mobilität der Beine sowie die Koordination „neuer“ Bewegungsabläufe im Vordergrund. Zu Letzteren zählt u. a. das Drehen im Bett in Seit- und Bauchlage. Es wird vermittelt, wie bei stabilem Rumpf mit mobilen Armen und Beine die Rotation en bloc erfolgen kann.

Aufsitzen im Bett. Es erfolgt mit stabilisiertem Rumpf über die Seitlage. Die Höhe des Betts ist so eingestellt, dass beim Sitz an der Bettkante die Beugung in den Hüftgelenken $70^{\circ}$ nicht übersteigt, um eine Flexion der Wirbelsäule zu vermeiden.

Sitzen. Bei den Sitzgelegenheiten im Zimmer und der Stationsumgebung ist auf ausreichende Höhe zu achten. Keilkissen erleichtern die Beckenaufrichtung und die Positionierung der Wirbelsäule in Aufrichtung.

Bücken. Hier lassen sich der horizontale und der vertikale Bücktyp unterscheiden. Er wird individuell durch die unterschiedlichen Proportionen von Beinlänge zu Rumpflänge vorgegeben. Der vertikale Bücktyp überwindet beispielsweise die Höhe mit aufrechter Wirbelsäule durch die Flexion in den Beingelenken. Dieses Bückverhalten ist für diesen Bücktyp nicht nur postoperativ, sondern generell das wirbelsäulenschonende Verhalten.

Heben und Tragen. Als Regel werden das Aufheben vom Boden über den 1-BeinKnie-Stand, das Heranführen der Last an den Rumpf und der Transport mit dem Lastgewicht im Rumpfkontakt geschult.

Die muskuläre Koordination, Kraft und Ausdauer werden kontinuierlich durch physiotherapeutische Methoden und Konzepte aufgebaut. Konzepte wie die propriozeptive neuromuskuläre Fazilitation (PNF), funktionelle Bewegungslehre (FBL), Spiraldynamik usw. arbeiten

Trauma Berufskrankh 2008 · 10 [Suppl 3]:408-410 DOI 10.1007/s10039-008-1412-y

(c) Springer Medizin Verlag 2008

\section{H. Belzl \\ Physiotherapie nach operativer Stabilisierung von Wirbelsäulenverletzungen}

\section{Zusammenfassung}

Die Physiotherapie nach operativer Wirbelsäulenstabilisierung verfolgt das Ziel, die aktive Aufrichtung, die posturale Steuerung und Beweglichkeit des Rumpfs und der Wirbelsäule wiederherzustellen. Die Funktionen sollen in Alltagsbewegungen integriert und die Selbstständigkeit des Patienten wieder erreicht werden. Hierzu ist eine detaillierte Behandlungsplanung auf der Basis umfassender Informationen des Operateurs sowie der physiotherapeutischen Befunderhebung erforderlich. Trotz Behandlungspfaden sind diese individuellen Gesichtspunkte Basis einer gezielten, funktionsbezogenen und schonenden Physiotherapie. Nach der physiotherapeutischen Behandlung in der stationären
Akutphase von 1-2 Wochen folgt eine Phase mit ambulanter Physiotherapie von der 3.-8. Woche. An diese können sich eine erweiterte ambulante Physiotherapie (EAP) oder eine berufsgenossenschaftliche stationäre Weiterbehandlung (BGSW) anschließen. Meist kann ab der 13. Woche eine Arbeitserprobung angedacht werden. Voraussetzung für eine effektive Therapie ist eine gemeinsame Strategie der Behandlung zwischen Operateur und Physiotherapeut.

\section{Schlüsselwörter}

Operative Wirbelsäulenstabilisierung · Physiotherapie · Behandlungsplanung · Behandlungspfad · Interdisziplinäre Zusammenarbeit

\section{Physiotherapy following surgical stabilization of spinal injuries}

\section{Abstract}

The aim of physiotherapy following spinal stabilization is to restore active straightening, posture control, as well as trunk and spinal mobility. The functions should be integrated in everyday movements and patient independence should be regained. To this end, a detailed treatment plan based on comprehensive information from the surgeon, as well as physiotherapeutic findings, is required. In spite of treatment pathways, these individual principles form the basis of a targeted, function-specific and sparing physiotherapy. The 1- to 2-week period of physiotherapeutic treatment during the acute in- patient phase is followed by a 3- to 8-week phase of out-patient physiotherapy. This can be followed by further out-patient physiotherapy or an approved in-patient follow-up treatment. Generally, a trial return to work can be considered from week 13 . A prerequisite of effective treatment is a joint treatment strategy between surgeon and physiotherapist.

\section{Keywords}

Surgical spinal stabilization - Physiotherapy . Therapy planning $\cdot$ Treatment pathways . Interdisciplinary cooperation 
stets aktiv, können verletzungsfern beginnen und enthalten Elemente der Lokomotion.

\section{Verlauf}

Der Rehabilitationsverlauf lässt sich am Beispiel berufsgenossenschaftlich Versicherter gut darstellen.

Auf eine stationäre Akutphase von 1-2 Wochen mit Physiotherapie, Anleitung zum wirbelsäulenschonenden Bewegungsverhalten und zu Eigenaktivität folgt eine Phase mit ambulanter Physiotherapie von der 3.-8. Woche.

Eine erweiterte ambulante Physiotherapie (EAP) kann sich für die 9.-12. Woche anschließen. Sie kombiniert Elemente der Physiotherapie, physikalischen Therapie und des medizinischen Aufbautrainings.

Alternativ gibt es die Möglichkeit zur berufsgenossenschaftlichen stationären Weiterbehandlung (BGSW). Sie hat einen noch höheren Therapieanteil pro Tag und wird um eine arbeitsplatzspezifische Ergotherapie erweitert.

In der Regel kann ab der 13. Woche eine Arbeitserprobung angedacht werden.

Innerhalb der Rehabilitationsphase (EAP, BGSW) liegt die physiotherapeutische Zielsetzung bei:

- Ausbau der dynamischen Stabilisation

- Arbeiten in Koordinationskomplexen

- Selbstständigkeit in Alltagsbewegungen

- Vorbereitung auf die beruflichen Anforderungen

Ein breites Spektrum physiotherapeutischer Techniken, Methoden und Konzepte kommt dabei zum Einsatz. Abhängig vom individuellen Ausbildungsstand und gemäß den Zulassungsbedingungen für die Durchführung der EAP/BGSW kommen PNF, FBL, manuelle Therapie, die Vojta-Methode oder Spiraldynamik zum Einsatz.

Die Eigenaktivität des Patienten wird in der täglichen Dauer in höherem Umfang eingefordert. Unterstützend erfolgen Behandlungen in der Gruppe in der Sporthalle oder im Bewegungsbad. Muskuläre Defizit werden im medizinischen
Aufbautraining mit einem persönlichen Trainingsplan gemindert.

\section{Fazit}

Bei einem derartigen umfassenden Therapiekonzept mit klaren Abläufen ist mit einer raschen und nachhaltigen Integration in Alltag, Freizeitsport und Beruf zu rechnen.

\section{Korrespondenzadresse}

\section{H. Belzl}

Schule für Physiotherapie/

Abteilung Physiotherapie,

BG Unfallklinik Tübingen,

Schnarrenbergstraße 95, 72076 Tübingen

hbelzl@bgu-tuebingen.de

Interessenkonflikt. Der korrespondierende Autor gibt an, dass kein Interessenkonflikt besteht.

\section{Literatur}

1. Ekkernkamp A, Schmidt J (2007) Standards für Heilverfahren und Rehabilitation. VBG, Hamburg

2. Hüter-Becker A, Dölken M (Hrsg) (2005) Physiotherapie in der Traumatologie/Chirurgie. Thieme, Stuttgart New York

3. Hüter-Becker A, Dölken M (Hrsg) (2005) Behandeln in der Physiotherapie. Thieme, Stuttgart New York

4. Wülker N (Hrsg) (2005) Orthopädie und Unfallchirurgie. Thieme, Stuttgart New York 